\title{
Effects of the Mycelial Extract of Cultured Cordyceps Sinensis on In Vivo Hepatic Energy Metabolism in the Mouse
}

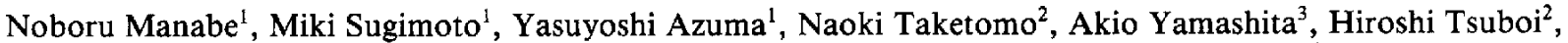 \\ Akinobu Tsunoo ${ }^{2}$, Noriko Kinjo ${ }^{4}$, Huang Nian-Lai ${ }^{5}$ and Hajime Miyamoto ${ }^{1}$ \\ 'Department of Animal Science, Faculty of Agriculture, Kyoto University, Kyoto 606-01, Japan \\ ${ }^{2}$ Meiji Institute of Health Science, Meiji Milk Products Co., Ltd., Odawara 250, Japan \\ ${ }^{3}$ Meiji Cell Technology Center, Meiji Milk Products Co., Ltd, Odawara 250, Japan \\ ${ }^{4}$ Department of General Education, Tokyo Medical and Dental University, Ichikawa 272, Japan \\ ${ }^{5}$ Sanming Mycological Institute, Fujian, People's Republic of China \\ Received August 24, 1995 Accepted November 10, 1995
}

\begin{abstract}
Mice were given the extract of cultured Cordyceps sinensis (Cs) ( $200 \mathrm{mg} / \mathrm{kg}$ daily, p.o.) for 3 weeks. In vivo phosphorus-31 nuclear magnetic resonance (NMR) spectra of the liver were acquired at weekly intervals using a surface coil. From 1 to 3 weeks, a consistent increase in the ATP/inorganic phosphate ratio, which represents the high energy state, was observed in the Cs extract-treated mice. The intracellular $\mathrm{pH}$ of the Cs extract-treated mice was not significantly different from that of the control mice. No steatosis, necrosis, inflammation or fibrosis were observed in the liver specimens from Cs extract-treated mice.
\end{abstract}

Keywords: Liver energy state, Phosphorus-31 nuclear magnetic resonance (NMR) (in vivo)

Cordyceps sinensis (Cs) is parasitic on the larvae of Lepidoptera. The fruit bodies of Cs together with the larvae have been used for a long time in traditional Chinese medicine. Various pharmacological effects of this Chinese medicine on animals both in vitro and in vivo have been reported $(1-4)$. These include in vivo antitumor activities $(1,2)$, inotropic effects on the left atrium (3), a cure for tuberculosis and a restorative action after various diseases (4). Previous in vitro studies have shown that the mycelial extract of cultured Cs produces non-purinergic and nonadrenergic inhibition of tracheal twitch contractions and relaxes the persistent contraction of the trachea and the aorta and that the Cs extract was more effective in reducing tracheal activities than the Chinese medicine (5). In the present study, we studied normal mice and mice with repeated oral administration of the Cs extract, with the aim of improving our understanding of the in vivo effects of the Cs extract on the ratios of hepatic phosphorylated metabolites noninvasively.

The hot water extract from Cs was prepared according to the method described in our previous report (5). Briefly, the strain SMIH8819 of Cs isolated at the Sanming Mycological Institute (Fujian, China) was cultured. The mycelia of the cultured Cs were then extracted with hot water $\left(90^{\circ} \mathrm{C}\right)$ for $2 \mathrm{hr}$. The extract was filtered through a membrane filter $(0.22 \mu \mathrm{m})$ and lyophilized. Male ddY mice (SLC, Shizuoka) weighing an average of $30.2 \mathrm{~g}$ at the beginning of the experiment were used at 6 weeks of age. During the experiment, the mice were given free access to tap water and a standard diet (CE-2; Clea Co., Ltd., Tokyo) in an air-conditioned room $\left(22 \pm 2{ }^{\circ} \mathrm{C}, \mathrm{RH}\right.$ $55 \pm 5 \%$ ) under a controlled light/dark cycle (12L/12D). All animals received humane care as outlined in the "Guide for the Care and Use of Laboratory Animals" (Kyoto University Animal Care Committee, according to NIH \#80-23, revised 1978). The animals were divided randomly into nine groups (six mice in each group). In groups V1, V2, V3 and V4 (vehicle control groups), the mice were given distilled water orally $(5 \mathrm{ml} / \mathrm{kg} /$ day) for 1 , 2,3 and 3 weeks, respectively. In groups Cs1, Cs2, Cs3 and Cs4 (Cs extract-treated groups), the animals received the $\mathrm{Cs}$ extract dissolved in distilled water orally at a dose of $200 \mathrm{mg} / \mathrm{kg} /$ day in volume of $5 \mathrm{ml} / \mathrm{kg}$ for $1,2,3$ and 3 weeks, respectively. In groups $\mathrm{V} 4$ and $\mathrm{Cs} 4$, the animals were kept without any treatment for 1 week after the 3-week administration period. Before treatment, six animals (V0 group) were killed under ether anesthesia. In groups V1, V2, V3, Cs1, Cs2 and Cs3, the animals were 
killed $2 \mathrm{hr}$ after the final administration, and those in groups V4 and Cs4 were killed $1 \mathrm{hr}$ after the final nuclear magnetic resonance (NMR) experiment. After the liver weight was recorded, each animal's liver was fixed in $10 \%$ formalin solution, embedded in paraffin, sectioned and stained with hematoxylin and eosin. The liver pathological results were observed by light microscopy and scored according to the method of Takahashi et al. (6).

NMR experiments were carried out using groups V4 and Cs4 at an interval of one week. In ${ }^{31} \mathrm{P}$ NMR examination, each mouse was anesthetized by intraperitoneal injection of sodium pentobarbital $(50 \mathrm{mg} / \mathrm{kg})$. During the NMR examination, the mouse was maintained in a probechamber at controlled air temperature of $25^{\circ} \mathrm{C}$. A modification of the method of Takahashi et al. (6) was used to obtain in vivo ${ }^{31} \mathrm{P}$ NMR spectra of the steady-state livers within intact animals. Briefly, ${ }^{31} \mathrm{P}$ NMR spectra were obtained using a JNM-alpha-400 FT-NMR spectrometer (JEOL, Tokyo) equipped with a $89-\mathrm{mm}$ bore super-conducting magnet (9.20 Tesla; Oxford Instruments, Bedford, MA, USA). The animal was mounted on a surface coil probe (two-turns, $2.0 \mathrm{~cm}$ diameter; JEOL) which was tuned to ${ }^{31} \mathrm{P}$ at $161.7 \mathrm{MHz}$ and used in conjunction with a ferrite screen composed of strips of computer tape (Scotch 700; Three M Co., St. Louis, MO) to eliminate ${ }^{31} \mathrm{P}$ NMR signals arising from superficial muscles (7). Field homogeneity was controlled by adjusting shim parameters using a water ${ }^{1} \mathrm{H}$ signal derived from the liver sample. Acquisition conditions were set as follows to obtain fully relaxed signals: the pulse width was $20 \mu \mathrm{sec}$, the pulse repetition time was $3.0 \mathrm{sec}$, and $200 \mathrm{scans}$ were accumulated. Thus, it took $10 \mathrm{~min}$ for each measurement. After exponential multiplication, the accumulated free induction decays were subjected to Fourier transformation. Individual spectral peak areas were calculated by computer integration with JEOL software (JEOL), and the creatine phosphate $(\mathrm{CP})$ peak was used as a positional reference $(0 \mathrm{ppm})$. The relative amount of each compound was converted to absolute concentration by comparison with the data of an external reference $(20 \mathrm{mM}$ methylene diphosphonate in water) in a spherical bulb. The relative ATP levels were obtained by taking the ratio of the $\beta$-phosphate peak of ATP ( $\beta$-ATP) to the reference peak (8), and then the $\beta$-ATP/inorganic phosphate peak area ratio ( $\beta$-ATP/Pi ratio) was calculated to evaluate in vivo liver energy metabolism. The intracellular $\mathrm{pH}$ was determined from the chemical shift difference between the Pi peak and the $\alpha$-phosphate peak of ATP (9). All NMR experiments were performed at $25 \pm 1{ }^{\circ} \mathrm{C}$.

The significance of differences between Cs extracttreated and the vehicle control mice was assessed by Student's paired $t$-test carried out with the StatView IV program using a Macintosh computer. Differences at a probability of $\mathrm{P}<0.05$ were considered significant. All data are reported as means \pm S.E.

The Cs extract had no effect on weight gain, liver weight or relative liver weight (Table 1). Moreover, no liver specimens obtained from either Cs extract-treated or the vehicle control mice showed steatosis, necrosis, inflammation or fibrosis. These findings suggest that the Cs extract had no toxic effect on the mouse liver.

The use of in vivo ${ }^{3 t} \mathrm{P}$ NMR spectroscopy made it possible to overcome the sampling problem associated with conventional biochemical analysis, i.e., HPLC analysis, of liver extract, although it only measured free ATP and not total ATP. Previous studies showed that in vivo serial ${ }^{31} \mathrm{P}$ NMR spectroscopy is a useful technique to detect the changes in liver energy state induced by $\mathrm{CCl}_{4}$ administration in mice (7) and rats $(10,11)$ and chronic ethanol feeding in rats $(6,12)$. Figure 1 shows representative examples of the in vivo ${ }^{31} \mathrm{P}$ NMR spectra acquired from $\mathrm{Cs}$ extract-treated and vehicle control mice at 3 weeks (Fig.

Table 1. Changes in body weight, liver weight and relative liver weight in the Cs extract-treated and vehicle control mice

\begin{tabular}{|c|c|c|c|c|c|}
\hline & \multicolumn{5}{|c|}{ Duration (weeks) } \\
\hline & $0^{1)}$ & 1 & 2 & 3 & 4 \\
\hline \multicolumn{6}{|c|}{ Body weight (g) } \\
\hline Cs extract & - & $32.8 \pm 1.0$ & $33.8 \pm 0.8$ & $36.2 \pm 1.2$ & $38.8 \pm 1.5$ \\
\hline Control & $30.0 \pm 0.9$ & $32.7 \pm 0.8$ & $33.5 \pm 0.5$ & $36.2 \pm 1.0$ & $38.7 \pm 1.0$ \\
\hline \multicolumn{6}{|c|}{ Liver weight (mg) } \\
\hline Cs extract & - & $1508 \pm 38$ & $1558 \pm 38$ & $1575 \pm 61$ & $1692 \pm 66$ \\
\hline Control & $1375 \pm 52$ & $1475 \pm 27$ & $1525 \pm 42$ & $1533 \pm 52$ & $1683 \pm 52$ \\
\hline \multicolumn{6}{|c|}{ Relative liver weight $(\%)$} \\
\hline Cs extract & - & $4.59 \pm 0.07$ & $4.61 \pm 0.17$ & $4.35 \pm 0.07$ & $4.36 \pm 0.07$ \\
\hline Control & $4.53 \pm 0.09$ & $4.52 \pm 0.11$ & $4.55 \pm 0.09$ & $4.24 \pm 0.07$ & $4.35 \pm 0.02$ \\
\hline
\end{tabular}

Data are reported as means \pm S.E. of 6 animals. ${ }^{1)}$ Before treatment, mice were killed under ether anesthesia. 


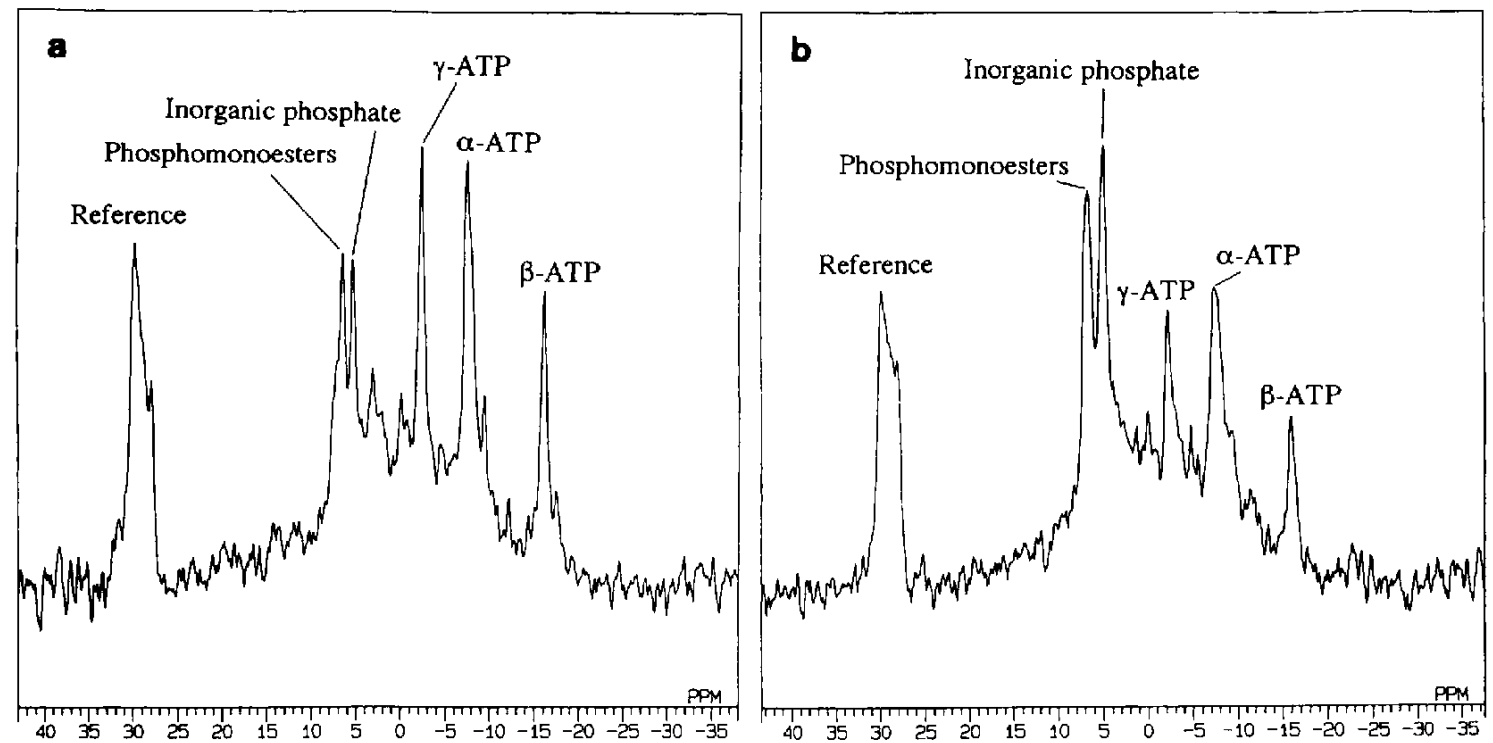

Fig. 1. In vivo ${ }^{31} \mathrm{P}$ NMR spectra obtained from the Cs extract-treated (a) and vehicle control mice (b) at 3 weeks after the initial administration. Prominent peaks observed were phosphomonoester, inorganic phosphate and nucleotide triphosphate (mainly $\gamma$-ATP, $\alpha$-ATP and $\beta$-ATP). The $\gamma$-ATP and $\alpha$-ATP signals may contain contributions from $\beta$ - and $\alpha$-ADP, respectively. The signal corresponding to $\beta$-ATP, which has no overlap with other phosphate signals, was used to quantify ATP. Increases in hepatic ATP levels and decreases in the inorganic phosphate levels were demonstrated in mice treated with Cs extract.

1: $a$ and $b$, respectively). Peak assignments are given in the legend. The spectral resolution was sufficient to measure individual peak areas using a digital integration technique. The resolution and the signal-to-noise ratio of the ${ }^{31} \mathrm{P}$ spectra obtained by the present in vivo method were sufficient for the quantitative evaluation of peak area ratios. This figure shows that the peak intensity of the $\beta$ ATP resonance was higher, but the peak intensity of the $P i$ resonance was lower in the Cs extract-treated mice than in the vehicle control mice.
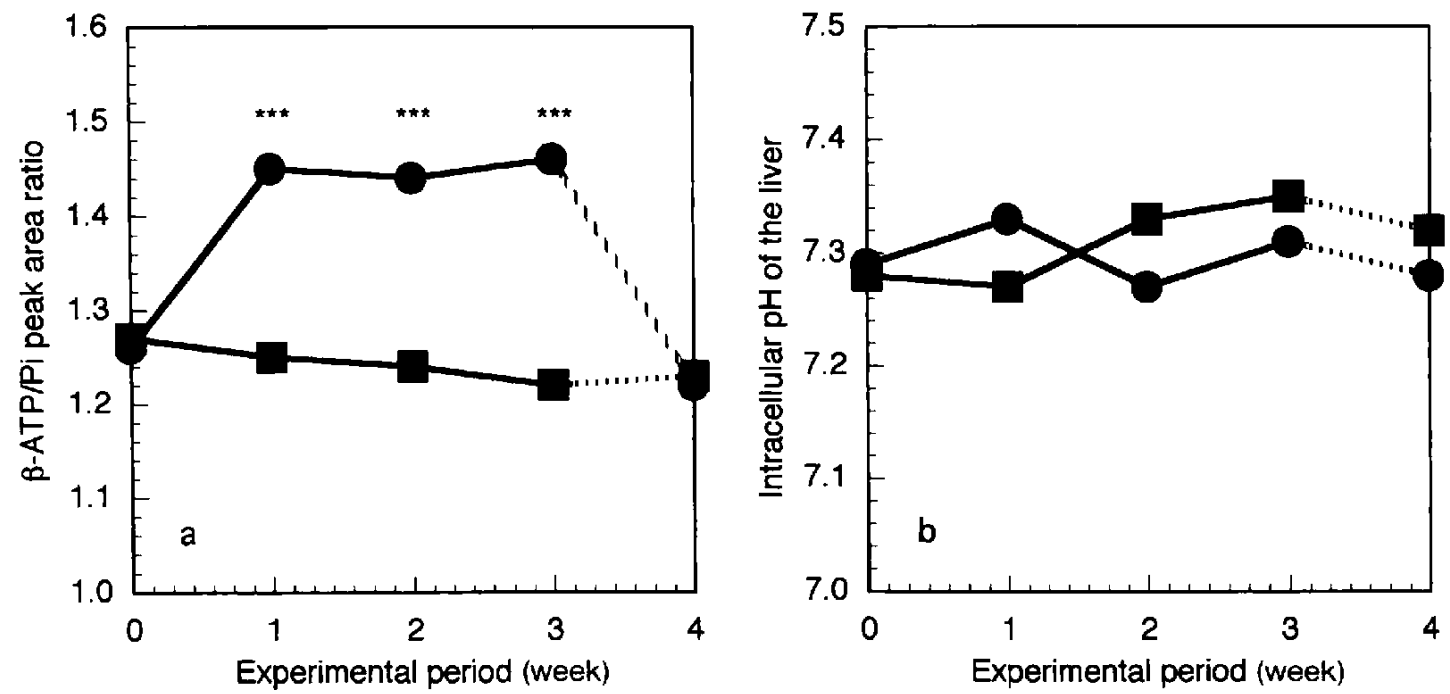

Fig. 2. Time course of the $\beta$-ATP/Pi ratio (a) and intracellular $\mathrm{pH}$ measured from the chemical shift of the Pi peak in ${ }^{31} \mathrm{P}$ NMR spectra (b) obtained from the liver in the Cs extract-treated and the vehicle control mice. The Cs extract-treated mice received the Cs extract dissolved in distilled water orally at a dose of $200 \mathrm{mg} / \mathrm{kg} /$ day in a volume of $5 \mathrm{ml} / \mathrm{kg}$ for 3 weeks, and the vehicle control mice were given distilled water orally $(5 \mathrm{ml} / \mathrm{kg} /$ day) for 3 weeks. Then the animals were kept without any treatment for 1 week after the 3-week administration period. $\square$ : Control, 0 : Cs extract. Each point represents the mean of 6 animals. ${ }^{* * *} \mathrm{P}<0.001$ vs. control. 
Figure 2a shows the time courses of the $\beta$-ATP/Pi peak area ratios obtained from the liver in the Cs extract-treated and the vehicle control mice, as measured by ${ }^{31} \mathrm{P}$ NMR spectroscopy. During the Cs extract administration period (from 0 to 3 weeks), the $\beta$-ATP/Pi ratios of the Cs extract-treated mice were higher as compared with those in the vehicle control mice $(P<0.05)$. One week after the final $\mathrm{Cs}$ administration, there were no significant differences in the $\beta-\mathrm{ATP} / \mathrm{Pi}$ ratios between these two groups. As the ADP level in the liver cannot be measured easily by in vivo ${ }^{31} \mathrm{P}$ NMR spectroscopy, we used the $\beta$-ATP/Pi ratio as an index of the hepatic energy state rather than the ATP/ADP ratio, according to Takahasi et al. (6). The increase in the $\beta$-ATP/Pi ratio suggests strongly that there was a higher energy state in the liver of the CS extract-treated mice than in the controls. The induction of ATP synthesis is caused by activated adenine translocase activity and/or mitochondrial respiratory chain function. The decrease in ATP consumption attributed to depressed sodium pump function can theoretically produce a high energy state in the liver (6). Like insulin (13), malotilate, which is a drug for liver cirrhosis, enhances protein synthesis in normal rat liver, facilitates the liver regeneration in cirrhotic rats, and activates the mitochondrial functions, which results in the increases of ATP concentration and adenylate energy charge in the liver of partially hepatectomized rats (14). The mechanism underlying the high hepatic energy state brought about during Cs extract administration, however, remains unclear. Further work is currently in progress in our laboratory to elucidate the mechanism.

The time course of changes in the intracellular $\mathrm{pH}$ of the liver measured by the chemical shift difference between the Pi and $\alpha$-ATP peaks in the ${ }^{31} \mathrm{P}$ NMR spectra are shown in Fig. 2b. The intracellular pH in the CS extracttreated mice was not significantly lower than that in the vehicle-treated controls throughout this study. The liver is markedly acidified under hypoxic conditions $(6,15)$. The lack of a shift in intracellular $\mathrm{pH}$ in this experiment might indicate normoxic conditions in the liver of the $\mathrm{Cs}$ extract-treated mice.

In summary, the present study showed that Cs extract induces a high energy state of the liver in mice and that there was no toxic effect of the Cs extract on mouse liver. These results suggest that the high energy state of the liver in the Cs extract-treated mice is a primary effect of repeated administration of the Cs extract.

\section{Acknowledgments}

We thank Mrs. K. Kuramitsu, Y. Kuribayashi and M. Miyamoto for excellent technical assistance. This work was supported in part by a Grant-in-Aid to N.M., M.S. and H.M. from the Ministry of Education, Science, Sports and Culture, Japan.

\section{REFERENCES}

1 Hamada M: Effect of Cordyceps sinensis (Berk.) Sacc. extract upon anti-tumor activity and tumor immunity. J Kanazawa Med Univ 16, 46-54 (1991)

2 Yoshida J, Takamura S, Suzuki S and Koshimura S: Potentiating effect of an extract of Cordyceps sinensis (Berk.) Sacc. on cytostatic activity of mouse effector cells against tumor cells. J Kanazawa Med Univ 17, 330-335 (1992)

3 Furuya $T$, Hirotani $M$ and Matsuzawa $M: N^{6}$-(2-Hydroxyethyl) adenosine, a biologically active compound from cultured mycelia of Cordyceps and Isaria species. Phytochemistry 22, $2509-2512$ (1983)

4 Chatterjee R, Srinivasan KS and Maiti PC: Cordyceps sinensis (Berkeley) Saccardo: Structure of Cordycepic acid. J Am Pharmaceut Assoc 66, 114-118 (1957)

5 Tsunoo A, Taketomo N, Kamijo M, Yamashita A, Kinjo N, and Huang N: Pharmacological effects of the mycelial extract of cultured Cordyceps sinensis on airways and aortae of the rat. In Mushroom Science, Vol XIV, Science and Cultivation of Edible Fungi, Edited by Elliott TJ, pp 425-431, Balkema, Rotterdam (1995)

6 Takahashi $\mathrm{H}$, Geoffrion Y, Butler KW and French SW: In vivo hepatic energy metabolism during the progression of alcoholic liver diseases: $A$ noninvassive ${ }^{31} \mathrm{P}$ nuclear magnetic resonance study in rats. Hepatology 11, 65-73 (1990)

7 Geoffrion Y, Rydzy M, Butler KW, Smith ICP and Jarrell HC: The use of immobilized ferrite to enhance the depth selectivity of in vivo surface coil NMR spectroscopy. NMR Biomed 1, $107-112$ (1988)

8 Azuma Y, Manabe N, Kawai F, Kanamori $M$ and Miyamoto $H$ : Phosphorus-31 nuclear magnetic resonance study of energy metabolism in intact slow- and fast-twitch muscles of rats. J Anim Sci 72, 103-108 (1994)

9 Malloy CR, Cunningham CC and Radda GK: The metabolic state of the rat liver in vivo measured by ${ }^{31}$ P-NMR spectroscopy. Biochim Biophys Acta 885, 1-11 (1986)

10 Bates TE, Williams SR, Busza AL and Gadian DG: A ${ }^{31} \mathbf{P}$ nuclear magnetic resonance study in vivo of metabolic abnormalities in rats with acute liver failure. NMR Biomed 1, 67-73 (1988)

11 Sandhu GS, Gonnella NC, Kapeghian JK, Plocinski AK, Plutchok J and Schlosser MJ: Evaluation of ${ }^{31} \mathrm{P}$ NMR spectroscopy as an indicator of chemically induced hepatic toxicity in the rat: Comparison with serum enzyme levels and pathology. NMR Biomed 4, 12-15 (1991)

12 Brauer $M$ and Ling M: The effect of chronic ethanol consumption on the intact rat liver studied by in vivo ${ }^{31} \mathrm{P}$ NMR spectroscopy. Magn Reson Med 20, 100-112 (1991)

13 Ozawa $K$, Yamaoka $Y$, Nannbu $H$ and Honjo I: Insulin as the primary factor governing changes in mitochondrial metabolism leading to liver regeneration and atrophy. Am J Surg 127, $669-675$ (1974)

14 Niwano $\mathrm{Y}$, Konaka $S$, Uchida $M$ and Sugimoto T: Activation of mitochondrial functions by malotilate in relation to accelerated liver regeneration in partial hepatectomized rats. Jpn J Pharmacol 42, 525-529 (1986)

15 Desmoulin F, Canioni P, Crotte C, Gérolami A and Cozzone PJ: Hepatic metabolism during acute ethanol administration: A phosphorus-31 nuclear magnetic resonance study on the perfused rat liver under normoxic or hypoxic conditions. Hepatology 7, 315-327 (1987) 